\title{
Interaction between the atmospheric boundary layer and a stand- alone wind turbine in Gansu-Part II: Numerical analysis
}

\author{
Zhi Zheng ${ }^{1,2,3 \dagger}$, ZhiTeng Gao ${ }^{1 \dagger \dagger}$, DeShun $\mathrm{Li}^{1,2,3}$, RenNian $\mathrm{Li}^{1,2,3^{*}}, \mathrm{Ye} \mathrm{Li}^{4^{*}}$, \\ QiuHao $\mathrm{Hu}^{4}$, and WenRui $\mathrm{Hu}^{5 *}$ \\ ${ }^{1}$ School of Energy and Power Engineering, Lanzhou University of Technology, Lanzhou 730050, China; \\ ${ }^{2}$ Gansu Provincial Technology Centre for Wind Turbines, Lanzhou 730050, China; \\ ${ }^{3}$ Key Laboratory of Fluid Machinery and Systems, Lanzhou 730050, China; \\ ${ }^{4}$ School of Naval Architecture, Ocean and Civil Engineering, Shanghai Jiao Tong University, Shanghai 200240, China; \\ ${ }^{5}$ Institute of Mechanics, Chinese Academy of Sciences, Beijing 100080, China
}

Received February 10, 2018; accepted March 28, 2018; published online May 3, 2018

\begin{abstract}
To analyze the interaction between wind turbines and the atmospheric boundary layer, we integrated a large-eddy simulation with an actuator line model and examined the characteristics of wind-turbine loads and wakes with reference to a corresponding experiment in Gansu. In the simulation, we set the wind turbine to have a rotor diameter of $14.8 \mathrm{~m}$ and a tower height of $15.4 \mathrm{~m}$ in the center of an atmospheric boundary layer with a $10.6^{\circ}$ yaw angle. The results reveal an obviously skewed wake structure behind the rotor due to the thrust component normal to the flow direction. The power spectra of the inflow fluctuation velocity exhibit a region of $-5 / 3$ slope, which confirms the ability of large-eddy simulations to reproduce the energy cascade from larger to smaller scales. We found there to be more energy in the power spectrum of the axial velocity, which shows that coherent turbulence structures have more energy in the horizontal direction. By the conjoint analysis of atmospheric turbulence and windturbine loads, we found that when the inflow wind direction changes rapidly, the turbulence kinetic energy and coherent turbulence kinetic energy in the atmospheric turbulence increase, which in turn causes fluctuations in the wind turbine load. Furthermore, anisotropic atmospheric turbulence causes an asymmetric load cycle, which imposes a strike by the turbine blade on the shaft, thereby increasing the fatigue load on the shaft. Our main conclusion is that the atmospheric boundary layer has a strong effect on the evolution of the wake and the structural response of the turbine.
\end{abstract}

wind power, atmospheric turbulence effects, finite volume methods, large-eddy simulations

PACS number(s): $89.30 . \mathrm{Ee}, 42.68 . \mathrm{Bz}$, 47.11.Df, 47.27.ep

Citation: Z. Zheng, Z. T. Gao, D. S. Li, R. N. Li, Y. Li, Q. H. Hu, and W. R. Hu, Interaction between the atmospheric boundary layer and a stand-alone wind turbine in Gansu-Part II: Numerical analysis, Sci. China-Phys. Mech. Astron. 61, 094712 (2018), https://doi.org/10.1007/s11433-018-9214-1

\section{Introduction}

The atmospheric boundary layer (ABL) is the most im-

\footnotetext{
$\uparrow$ These authors contributed equally to this work.

*Corresponding authors (RenNian Li, email: lirn@lut.cn; Ye Li, email: ye.li@sjtu.

edu.cn; WenRui Hu, email: wrhu@imech.ac.cn)
}

portant atmospheric component in wind-power applications. Research on both the ABL and wind turbines has been conducted for decades, but their interaction has not been well studied. Studies of the impact of wind turbines on the atmosphere have mainly focused on the reduction of wind speed and the influence of the vertical turbulence structure [1-5]. The main conclusion has been that wind turbines have 
a strong effect on the vertical turbulent fluxes of momentum and heat in the local meteorology, but that impacts on natural climate interannual variability are extremely limited $[1,6]$. The impact of the atmosphere on wind energy utilization is more complicated and powerful. The movement of the atmosphere produces various coherent turbulent structures, which lead to complex inflow conditions. Chamorro et al. [710] performed a series of wind tunnel tests and numerical experiments on a miniature wind turbine, which was subjected to both neutral and stable boundary layers. Their studies showed that a stronger wind shear of the incoming flow in the stable boundary layers leads to a stronger and larger region of enhanced turbulence intensity and the faster recovery of velocity in the near-wake region. Atmospheric turbulence also has important implications for the structural response and wake evolution of turbines. Churchfield et al. [11] used large-eddy simulation to explore the effects of atmospheric stability and surface roughness on wind turbine dynamics. The authors observed high or low peak-to-peak high-frequency responses in the structural moments of the turbine, which are caused by the turbine's interaction with coherent turbulent structures. España et al. [12] reported the phenomenon of wake meandering throughout an experiment in an ABL wind tunnel. Recent experiments and numerical simulations [13-15] have shown that wake meandering is enhanced in the presence of geostrophic forcing. The operational responses of a wind turbine to the atmosphere influence the wake evolution of the turbine, which also point to the possibility of developing advanced turbine controls for strategically manipulating wake dynamics to enable overall wind farm optimization [16]. Some researchers [17-19] have proposed increasing the total power output of wind farms by installing an upstream turbine, which extracts less power than subsequent turbines, and operating the upstream turbine in a yawed condition. It is evident that studies of the interaction between the atmosphere and wind turbines have become important in both the wind energy and atmospheric science communities.

In numerical simulations of turbulence, the main methods used are Reynolds-averaged Navier-Stokes (RANS), largeeddy simulation (LES), and direct numerical simulation (DNS) [20-22]. The ABL has a high-Reynolds-number turbulent boundary-layer flow $\left(\operatorname{Re} O\left(10^{6}\right)\right)$ [23] and DNS requires too many meshes and is not suitable for simulating ABL with a wind turbine. Although the computational efficiency of RANS is superior, it has some shortcomings in resolving complex transient flow problems such as underestimating the loss of wake speed, delaying wake recovery, and reducing the wake radius [24]. As an intermediate approximation between DNS and RANS, LES can provide high-resolution spatial and temporal information regarding the ABL and wind turbines. Recently, researchers have applied LES to wind-turbine wakes [25-27]. For wind-turbine simulations, the main methods used are the actuator line model (ALM) [28,29] and actuator disk model (ADM) [30,31]. Compared to the ADM, the advantages of the ALM are that it contains more physical mechanisms of the rotary aerodynamics in wind-turbine wakes, and it has proven to be accurate for most flow conditions and rotor configurations $[18,32,33]$.

Based on field experiments conducted in Gansu, which we describe in sect. 2 , in this study, we primarily analyze the characteristics of wind-turbine loads and wakes in the ABL. We used LES to generate turbulent inflow atmospheric winds and ALM to model the body forces exerted by the wind turbine, which we describe in sect. 3 . We present and discuss the LES results in sect. 4 and draw our conclusions in sect. 5.

\section{Experiment setup}

In the experimental research, we used a 33-kW 2-blade wind turbine with a rotor diameter, $D$, of $14.8 \mathrm{~m}$ and a hub height of $15.4 \mathrm{~m}$. The turbine reaches a rated power of $33 \mathrm{~kW}$ at a wind speed of $11 \mathrm{~m} \mathrm{~s}^{-1}$ and a rotational speed of $85 \mathrm{r} / \mathrm{min}$. The blade uses NACA44 series airfoils.

As shown in Figure 1(a), we performed wake measurement

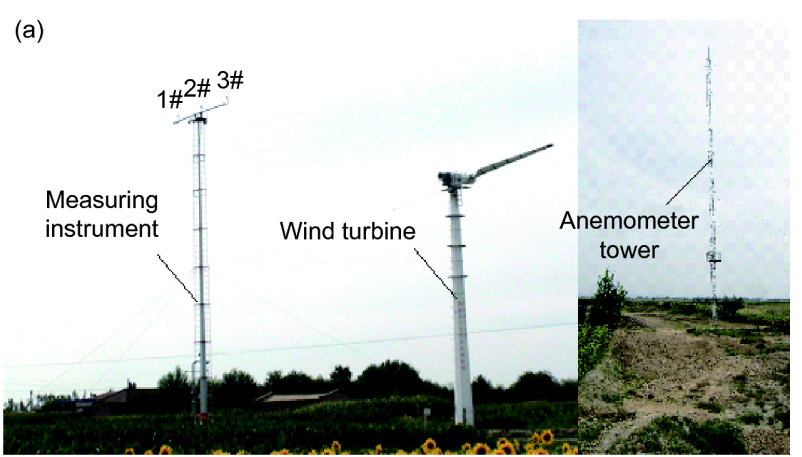

(b)

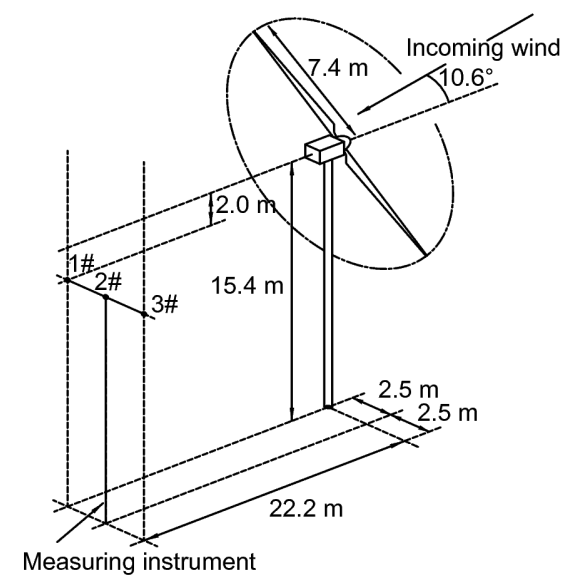

Figure 1 (Color online) Experimental setup for the wake measurement. (a) Side view of the wind turbine (right) and measuring instrument (left). (b) Schematic of the setup during deployment. 
on a flat ground area in Gansu, China $\left(37^{\circ} 05^{\prime} \mathrm{N}, 103^{\circ} 57^{\prime} \mathrm{E}\right)$. In the experimental setup, we placed a $30-\mathrm{m}$ guyed anemometer tower with three anemometers (at 8, 15.4, and $22.8 \mathrm{~m}$ height, respectively) in front of the wind turbine. As a primary reference for incoming wind, we placed ultrasonic wind speed and direction sensors with a sampling frequency of $5 / 3 \mathrm{~Hz}$ at hub height. The wake measurement instrument consists of an 18-m hydraulic lift, an anemometer installation platform, and three CSAT3 3D ultrasonic anemometers with a sampling frequency of $20 \mathrm{~Hz}$. As shown in Figure 1(b), wake measurements were performed at a distance of $1.5 \mathrm{D}$ from the wind turbine. At the top of the wake measurement instrument, the three ultrasonic anemometers are separated by $2.5 \mathrm{~m}$ at a distance of $2.0 \mathrm{~m}$ below the wind turbine axis. More details regarding the wake experiment can be found in the work of Li et al. [34].

Figure 2(a) shows the changes in the incoming wind velocity (Vel.), yaw angle, humidity (Hum.), and rotational speed $(n)$ at about $23.1{ }^{\circ} \mathrm{C}$ and $0.82 \mathrm{~atm}$. During the 6-s sampling time, the flow conditions and wind turbine operational states remain essentially unchanged, so we can assume that the turbine is in a steady state of a $4.02 \mathrm{~m} \mathrm{~s}^{-1}$ inflow with a $10.6^{\circ}$ yaw angle. In Figure 2(b), we can see that the time history of the wake flow velocity at measurement points $1 \#$, $2 \#$, and $3 \#$ remain unchanged throughout the sampling time.

\section{Numerical methods}

\subsection{Governing equations}

Eqs. (1) and (2) give the grid-filtered continuity and NavierStokes (N-S) equations for the incompressible LES, respectively, and eq. (3) gives the resolved potential temperature transport equation, where the overbar denotes a grid-scale filtering operation.

$\frac{\partial \bar{u}_{j}}{\partial x_{j}}=0$,

$$
\begin{aligned}
\frac{\partial \bar{u}_{i}}{\partial t}+\frac{\partial}{\partial x_{j}}\left(\bar{u}_{j} \bar{u}_{i}\right)= & -2 \varepsilon_{i 3 k} \Omega_{3} \bar{u}_{k}-\frac{\partial \widetilde{p}}{\partial x_{i}} \\
& -\frac{1}{\rho_{0}} \frac{\partial}{\partial x_{i}} \bar{p}_{0}(x, y)-\frac{\partial \tau_{i j}^{D}}{\partial x_{j}} \\
& -g\left(\frac{\bar{\theta}-\theta_{0}}{\theta_{0}}\right) \delta_{i 3}+\frac{1}{\rho_{0}} f_{i}^{\mathrm{T}} .
\end{aligned}
$$

From left to right, the terms in the N-S equation represent transient, convective, Coriolis, pressure, horizontal driving pressure, fluid stress tensor, buoyancy, and other densitynormalized forces exerted by the wind turbine, respectively. The rotation rate vector $\Omega_{3}$ is equal to $\omega[0, \cos \phi, \sin \phi]$, where $\omega$ is the planetary rotation rate and $\phi$ is the latitude. $\theta_{0}=300 \mathrm{~K}$ is the reference temperature and $\bar{\theta}$ is the resolved potential temperature.

$\frac{\partial \bar{\theta}}{\partial t}+\frac{\partial}{\partial x_{j}}\left(u_{j} \bar{\theta}\right)=-\frac{\partial q_{j}}{\partial x_{j}}$,

where $q_{j}$ represents the flux in the temperature by viscous and sub-grid scale (SGS) effects.

\subsection{Sub-grid scale (SGS) model}

In all of the flow fields, except at the lower surface (the stress at the lower surface is given by the boundary condition in sect. 3.3), the deviatoric aspect of the SGS stress tensor $\tau_{i j}{ }^{\mathrm{D}}$ is as follows:

$\tau_{i j}^{\mathrm{D}}=-2 v^{\mathrm{SGS}} S_{i j}$,

where $v^{\mathrm{SGS}}$ is the SGS viscosity and $\bar{S}_{i j}$ is the mean strain-rate

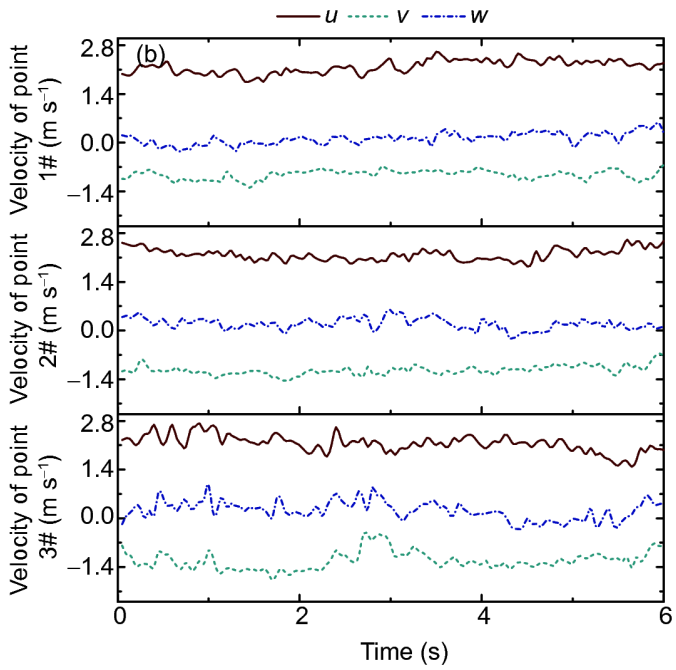

Figure 2 (Color online) Measurement results. (a) Incoming wind conditions and wind turbine operational status. (b) Time history of wake flow velocity at measurement points. $u$ is the component in the axial (rotor axis) direction, $v$ is the component in the lateral direction, and $w$ is the component in the vertical direction.

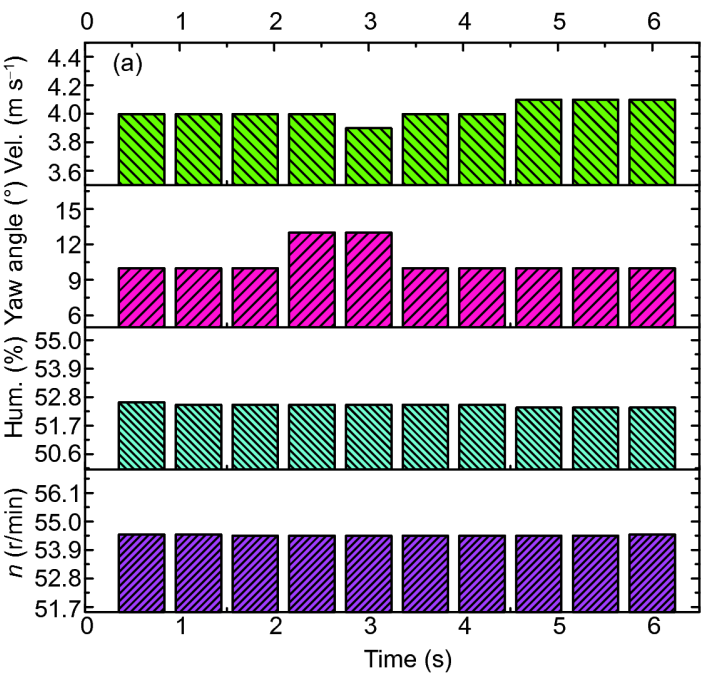


tensor, which are formulated using the standard Smagorinsky SGS model:

$v^{\mathrm{SGS}}=\left(C_{\mathrm{s}} \Delta\right)^{2}\left(2 \bar{S}_{i j} \bar{S}_{i j}\right)^{1 / 2}$,

$\bar{S}_{i j}=\frac{1}{2}\left(\frac{\partial \bar{u}_{i}}{\partial x_{j}}+\frac{\partial \bar{u}_{j}}{\partial x_{i}}\right)$.

$C_{\mathrm{s}}$ is the Smagorinsky constant, which is set to 0.168 , and $\Delta=V_{\text {grid }}^{1 / 3}$ is the SGS filter length scale, where $V_{\text {grid }}$ is the grid cell volume.

The temperature flux $q_{j}$ is as follows:

$q_{j}=-\frac{v^{\mathrm{SGS}}}{P r_{\mathrm{t}}} \frac{\partial \bar{\theta}}{\partial x_{j}}$,

where $P r_{\mathrm{t}}$ is the turbulent Prandtl number. For unstable and neutral conditions, $P r_{\mathrm{t}}$ is equal to $1 / 3$.

\subsection{Numerical setup}

In the simulation, we used a $3000 \mathrm{~m} \times 3000 \mathrm{~m} \times 1000 \mathrm{~m}$ physical domain in neutral atmospheric conditions with $4.02 \mathrm{~m} \mathrm{~s}^{-1}, 34.4^{\circ}$ north-by-east incoming wind speed. To determine the flow-field details near the wind turbines, we adopted five layers of mesh refinement in the simulation, as shown in Figure 3. The minimum mesh step size is about $0.5208 \mathrm{~m}$ and 28 grids are distributed across the rotor. Since the scale of the ABL simulation is on the order of kilometers, an acceptable grid scale in LES is on the order of meters, which leads to a limited bandwidth of the resolved physical quantities. Recently, Ghate and Lele [35] developed a physics-based modeling approach to significantly increase the spatial and temporal bandwidths in a cost-effective manner. This approach is attractive and consistent with the simulation
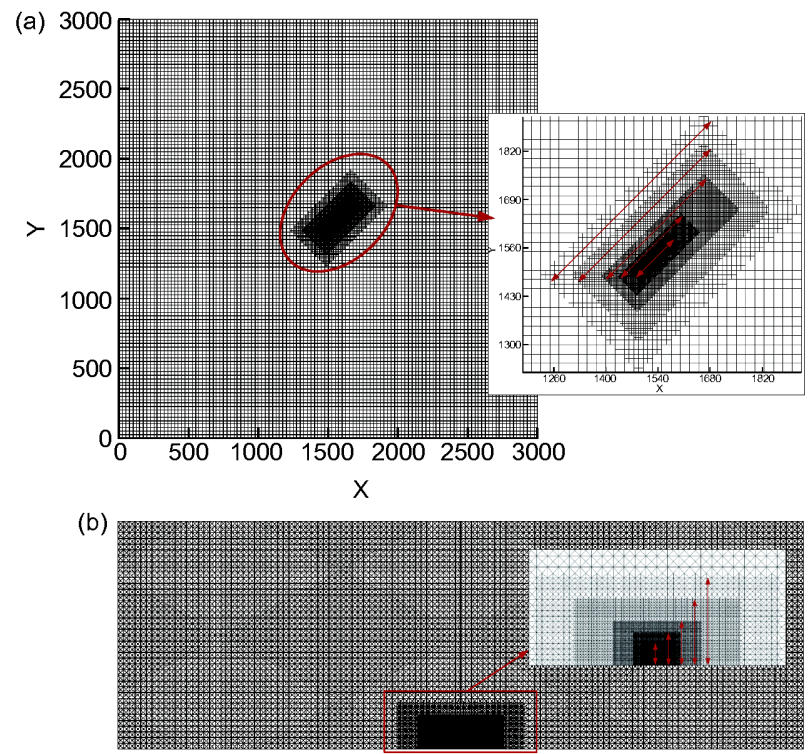

Figure 3 (Color online) Five layers of mesh refinement. (a) Top view of the mesh; (b) front view of the refinement field. strategy of wind farms, which will be considered in future work.

Under atmospheric turbulence with a high Reynolds number, the LES of a full-geometry wind turbine requires a significant amount of boundary-layer mesh, so we modeled the density-normalized forces in eq. (2) based on the concept of the actuator line. We determined the force projection following the work of Sørensen and Shen [32], as follows:

$f_{i}^{\mathrm{T}}(r)=\frac{F_{i}^{\mathrm{A}}}{\varepsilon^{3} \pi^{3 / 2}} \exp \left[-\left(\frac{r}{\varepsilon}\right)^{2}\right]$,

where $F_{i}^{\mathrm{A}}$ is the actuator element force, which is calculated using the blade element momentum (BEM) theory; $f_{i}^{\mathrm{T}}$ is the force field projected onto the grid in eq. (2), $r$ is the distance between the cell center and actuator point, and $\varepsilon$ controls the Gaussian width. The grid cell length $\Delta x$ is equal to 0.5208 near the actuator line, and it is better to keep $\varepsilon / \Delta x \approx 2$ as recommended by Troldborg [36], so we set $\varepsilon$ to 1 in this study.

At the lower surface, the deviatoric aspect of the stress tensor $\tau_{i j}^{\mathrm{D}}$ at the lower surface is given by the following:

$\tau_{i j}^{\mathrm{D}}=\left[\begin{array}{ccc}0 & 0 & \tau_{13}^{\mathrm{tot}} \\ 0 & 0 & \tau_{23}^{\mathrm{tot}} \\ \tau_{13}^{\mathrm{tot}} & \tau_{23}^{\mathrm{tot}} & 0\end{array}\right]$,

where $\tau_{13}^{\text {tot }}$ and $\tau_{23}^{\text {tot }}$ use Moeng's model [37]. The inputs to the models are the surface aerodynamic roughness height, $z_{0}$, which we set to $0.1 \mathrm{~m}$; the averaged value of the surface temperature flux, $q_{\mathrm{j}}$, which we set to 0 for neutral conditions; and the friction velocity, $u_{*}$, which is the square root of the magnitude of $\tau_{13}^{\text {tot }}$ and $\tau_{23}^{\text {tot }}$. We used the Monin-Obukhov similarity theory to estimate the friction velocity as follows:

$\frac{\left(\left\langle\bar{u}_{1 / 2}\right\rangle^{2}+\left\langle\bar{v}_{1 / 2}\right\rangle^{2}\right)^{1 / 2}}{u_{*}}=\frac{1}{\kappa} \ln \left(\frac{z}{z_{0}}+f(L)\right)$,

where $f(L)$ is the atmospheric stability-related function and $L$ is the Obukhov length. In neutral conditions, $f(L)$ is equal to 0 .

At the upper surface, we set the stress, temperature flux, and normal velocity to zero. The upper surface is above the entire boundary layer and any atmospheric turbulence, so these boundary conditions are appropriate. Since the stress and temperature flux are defined at the lower and upper surfaces thus far, there is no need to set boundary conditions for the temperature and horizontal velocity at these boundaries. All other boundaries are periodic for the precursor ABL simulation without any turbine. For the upstream boundaries of the simulation with the wind turbine, the velocity and temperature are specified by a space-varying boundary condition, drawn from the data planes saved from 
the precursor simulation at every time step once the flow reached a quasi-equilibrium state. At the downstream boundary, we set the gradients of velocity and temperature to allow the turbine wakes to exit without cycling back.

We discretized the models in sect. 3 using the central difference scheme in space and Crank-Nicolson scheme in time. To avoid pressure-velocity decoupling, we interpolated the velocity fluxes using the method of Rhie and Chow [38]. For the precursor ABL simulation, we used a variable timestep method to maintain a maximum Courant number of 0.75 . Initially, we simulated the precursor simulation for $30000 \mathrm{~s}$ to ensure that the quasi-equilibrium state is reached with an interval time of $2000 \mathrm{~s}$ to output the data. The actual result shows the appearance of an obvious turbulence structure at about $16000 \mathrm{~s}$. After $8000 \mathrm{~s}$, the sampled velocity oscillates at some mean value and we can consider the flow field to be in quasi-equilibrium. To make our data analysis more accurate, we performed the second numerical simulation for $50000 \mathrm{~s}$ with an interval time of $50 \mathrm{~s}$ to output the data, so we used the data between 30000 and $50000 \mathrm{~s}$ in our analysis of the ABL. In the simulation with the wind turbine, the velocity and temperature are specified by the boundary conditions taken from the data planes saved at $30050 \mathrm{~s}$. To capture the turbine and wake motions, we set the time step to $0.015 \mathrm{~s}$ to correspond to a blade rotation of $5^{\circ}$ and a maximum Courant number of about 0.24 , as recorded in the $\log$ file. The simulation times were $1200 \mathrm{~s}$ and we disregarded the first $600 \mathrm{~s}$ of data to ensure complete wake development. Therefore, the data used for the analysis of the wind turbine are from 30650 to $31250 \mathrm{~s}$.

\section{Results and discussion}

\subsection{Precursor ABL simulation}

Figure 4 shows the velocity magnitude on three sides of the geometric region, which reveals a distinct wind-shear phenomenon and high turbulence levels.

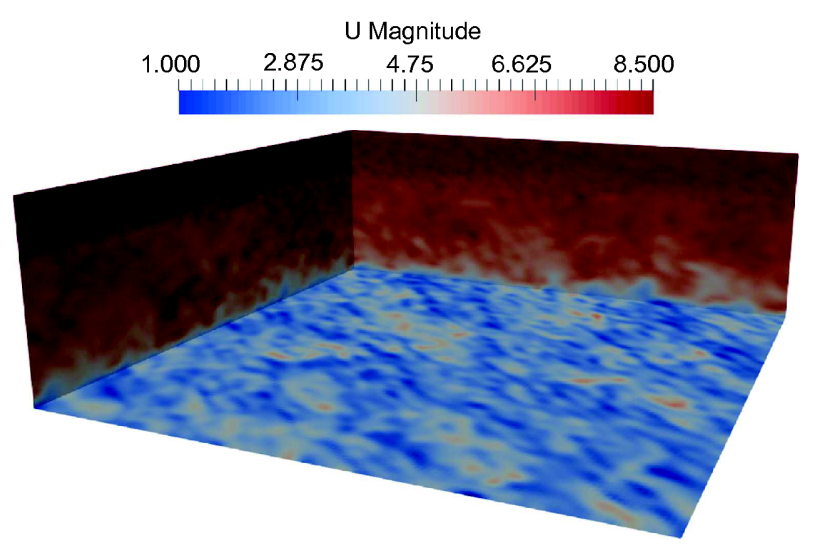

Figure 4 Wind speed on three sides of the geometric region (without wind turbines)
To further quantify the characteristics of the neutral atmosphere, we validated the neutral atmospheric characteristics of the boundary layer from three perspectives: the distribution of the potential temperature along the height, the average wind speed along the height, and the power spectra characteristics in fluctuating wind speeds.

Figure 5 shows the distribution of the potential temperature $\theta$ and rotor axial velocity $u$. The axial velocity $u$ obtained by LES is the average value between 30000 and $50000 \mathrm{~s}$, and is compared with the velocity given by the law of the wall (LOTW), which represents an undisturbed fully rough turbulent boundary layer. The axial velocity $u$ formulated by the LOTW is as follows:

$u=\frac{u_{*}}{\kappa} \ln \left(\frac{z}{z_{0}}\right)$,

where $u_{*}$ is the friction velocity, the von Karman constant $\kappa$ is 0.41 , and $z_{0}$ is the aerodynamic roughness length of $0.1 \mathrm{~m} . z^{+}$ is the normalized value of $z$, which is normalized by the rotor diameter $D$ and $u^{+}$is the normalized value of $u$, which is normalized by the wind speed $U_{0}$ at hub height. The potential temperature $\theta$ is constant $(300 \mathrm{~K})$ along the vertical direction (below $700 \mathrm{~m}$ ), which is the exact characteristic of a neutral ABL $(\partial \theta / \partial z=0)$. When $z^{+}>40, \theta$ increases as the height increases, which is characteristic of capping inversion. The velocity profile obtained by LES fits well with the LOTW near the ground $\left(1<z^{+}<20\right)$.

The power spectra of fluctuating wind speed should follow the improved von Karman spectrum, as recommended for neutral atmospheric conditions by the Engineering Sciences Data Unit (ESDU, originally the Technical Department of the Royal Aeronautical Society, owned since 1997 by IHS Inc.). To validate the fluctuating wind speed in neutral ABL conditions, we set a sample point in the center of the geometric region at a height of $15.4 \mathrm{~m}$. Figure 6 shows a comparison of the calculation spectrum of 3D fluctuating wind speed $(u, v, w)$ at the sample point as the improved von Karman spectrum. The result shows that the calculation spectrum agrees well with the target spectrum.
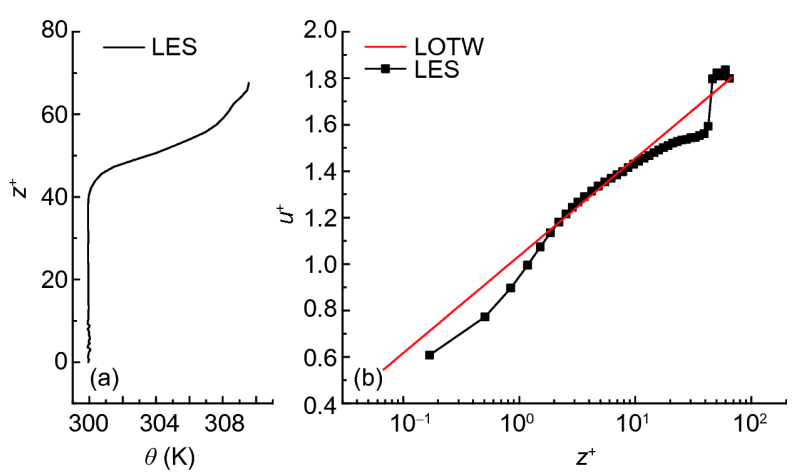

Figure 5 Distribution of the potential temperature (a) and rotor axial velocity (b). 

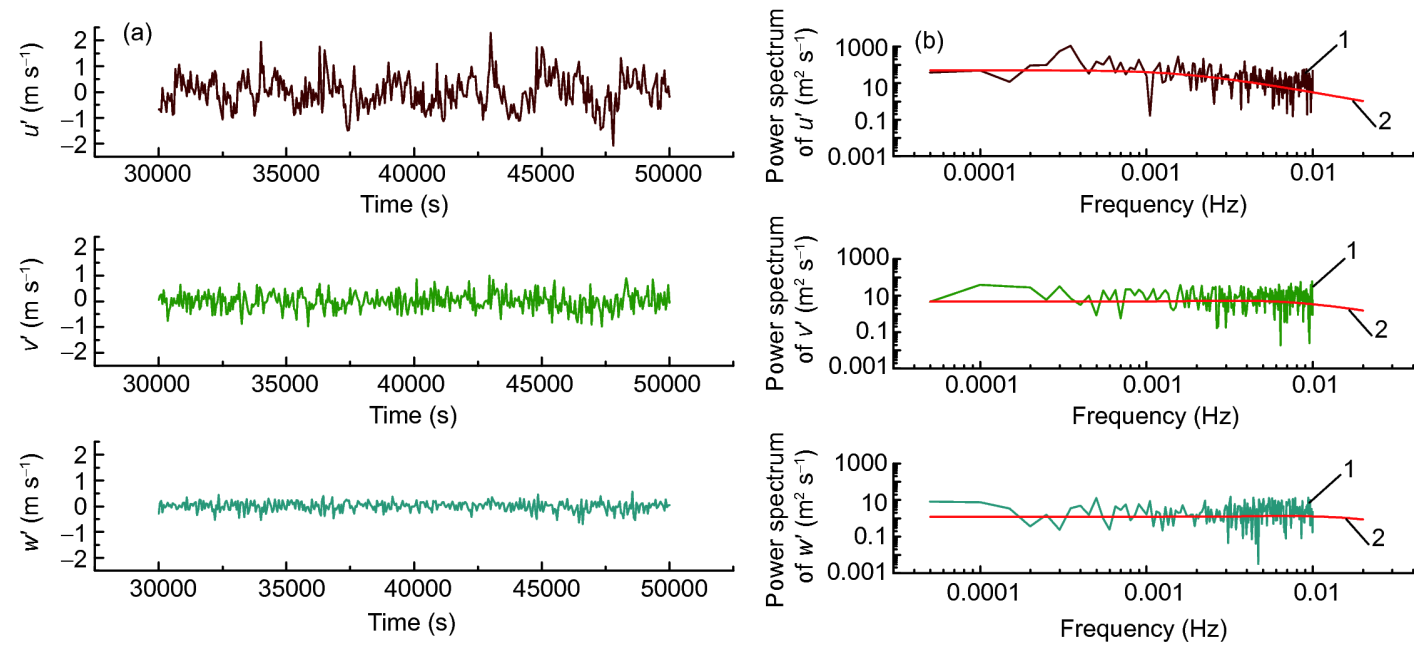

Figure 6 (Color online) Fluctuating wind speed and power spectra. (a) The fluctuating wind speed for $u, v$, and $w$ and (b) power spectra comparison between LES (1) and ESDU (2).

\subsection{Validation of the time-averaged velocity at mea- surement points}

As shown in Figure 7, we set a $33-\mathrm{kW}$ wind turbine in the center of the geometric region $(1500,1500,0)$ with a $10.6^{\circ}$ yaw angle to verify the accuracy of the neutral atmospheric wind plant calculation. We derived aerodynamic information about the NACA44 series airfoils with angles of attack ranging from $-180^{\circ}$ to $180^{\circ}$, as needed by the ALM, from ref. [39]. We used the Viterna-Corrigan model [40] to calculate the body force of the blades, based on the BEM theory.

Table 1 shows a comparison of results for the LES (average value in $600 \mathrm{~s}$ ) and available experimental data (average value in $6 \mathrm{~s}$ ) at measurement points $1 \#, 2 \#$, and $3 \#$. The calculated axial velocity $(u)$ results agree well with the experimental data at $1 \#$, but there are certain deviations at points $2 \#$ and $3 \#$. The main reason for this difference is that the numerical computation ignored the hub effect. Another

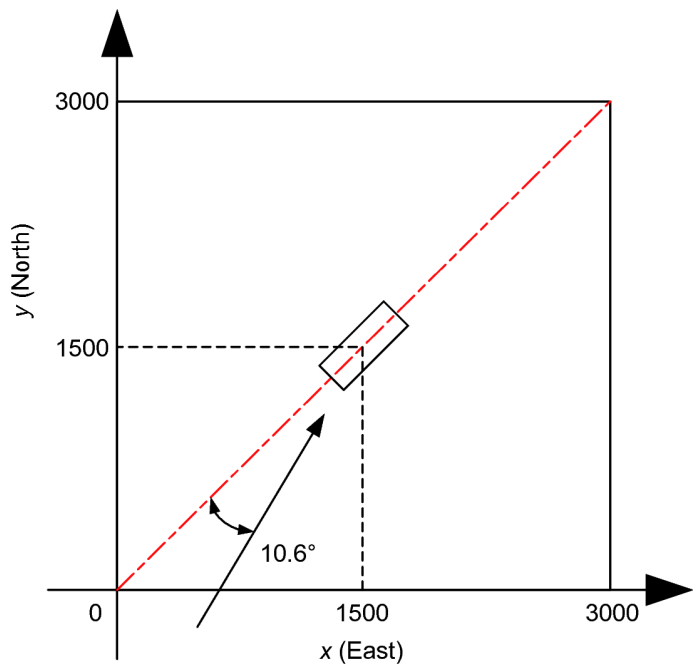

Figure 7 (Color online) Top view of the neutral wind plant.
Table 1 Comparison of LES (average value in $600 \mathrm{~s}$ ) and experiment (average value in $6 \mathrm{~s}$ ) results

\begin{tabular}{ccccc}
\hline \multirow{2}{*}{ Index } & \multicolumn{4}{c}{ Axial velocity $\left(\mathrm{m} \mathrm{s}^{-1}\right)$} \\
\cline { 2 - 5 } & $\begin{array}{c}-2 D \text { (in front of the } \\
\text { wind turbine) }\end{array}$ & $1 \#$ & $2 \#$ & $3 \#$ \\
\hline Experiment & 3.950 & 2.180 & 2.183 & 2.151 \\
LES & 4.136 & 2.207 & 2.820 & 2.836 \\
\hline
\end{tabular}

reason may be that the sampling period $(6 \mathrm{~s})$ of the experiment was not long enough to ensure adequate convergence of the statistical quantities.

Figure 8 shows a comparison of the distribution of velocity along the rotor axis in the far wake flow field by LES (solid line), the experimental value at measurement point $1 \#$ and the experimental value at the anemometer tower location in front of the wind turbine at a distance of $2 D$. As shown in Figure 8 , at about $2.5 \mathrm{D}$ distance behind the rotor, the wind speed dropped to a minimum and then gradually recovered.

\subsection{Power spectra of velocity components}

The auto-correlation function of the fluctuation velocity indicates whether the velocity at one moment has the same characteristics as the velocity at another moment delayed by $\tau$, and indirectly reveals the energy cascade effects with respect to time or frequency. According to the methods illustrated by Kundu and Cohen [41], we derived the autocorrelation function $R(\tau)$ of the fluctuation-velocity components from 40000 data items with a time step of $0.015 \mathrm{~s}$ in the 600 -s sampling period. To ensure the accuracy of the autocorrelation function, a sufficient amount of data must be used for averaging. We chose a maximum time delay of $300 \mathrm{~s}$ and used 19999 data items (data in $299.985 \mathrm{~s}$ ) for averaging. Figure 9 shows the auto-correlations of the axial fluctuation velocity $u^{\prime}$, lateral fluctuation velocity $v^{\prime}$, and vertical fluc- 


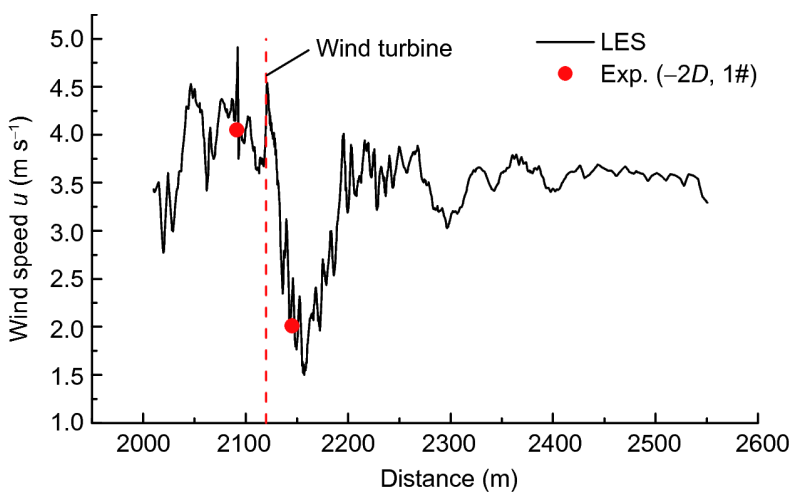

Figure 8 (Color online) Recovery trend of the velocity in the far wake flow.

tuation velocity $w^{\prime}$ at different locations. Overall, we found the correlations of $u^{\prime}$ to be greater than the correlations of $v^{\prime}$ and $w^{\prime}$. To compare different locations, the auto-correlation of the fluctuation velocity behind the rotor is larger. This is because the area in front of the wind turbine (e.g., $-2 D$ ) is mainly affected by atmospheric turbulence, and behind the rotor (e.g., $1.0 D, 1.5 D, 2.0 D$ ), the rotation of the wind turbine produces a strong and obvious vortex structure.

The fast Fourier transform (FFT) of the auto-correlation functions gives the power spectra of the fluctuation wind speeds. Figure 10 shows the power spectra of three fluctuation-velocity components in front of the wind turbine at a distance of $2 D$. As shown in Figure 10, all the spectra exhibit a region with a $-5 / 3$ slope (red line) to the filter cut off, which confirms LES's ability to reproduce the energy cascade from larger to smaller scales. Compared with those of $v^{\prime}$ and $w^{\prime}$, the power spectrum of $u^{\prime}$ contains more low-frequency energy, which indicates that coherent turbulent structures have more energy in the horizontal direction.

\subsection{Analysis of wake skew}

When the wind is not aligned with the axis of the wind turbine, the wake is skewed to one side of the turbine because the thrust is normal to the rotating plane, so it has a component normal to the flow direction. The force on the flow is therefore opposite to the thrust, which causes the flow to be skewed. The Coriolis force can also cause a skewed spatial structure and drive some of the turbulence energy away from the center of the wake. The center line of the wake will be at an angle $\chi$ to the axis of rotation, which is known as the wake skew angle. Coleman et al. [42] relates the angle $\chi$ to the yaw angle $\gamma$ in the following equation:

$\tan \chi=\frac{U_{0}\left(\sin \gamma-a \tan \frac{\chi}{2}\right)}{U_{0}(\cos \gamma-a)}$,

where $a$ is the axial induction factor. As shown in Figure 11(a), the axial induction factor $a$ in the wake flow
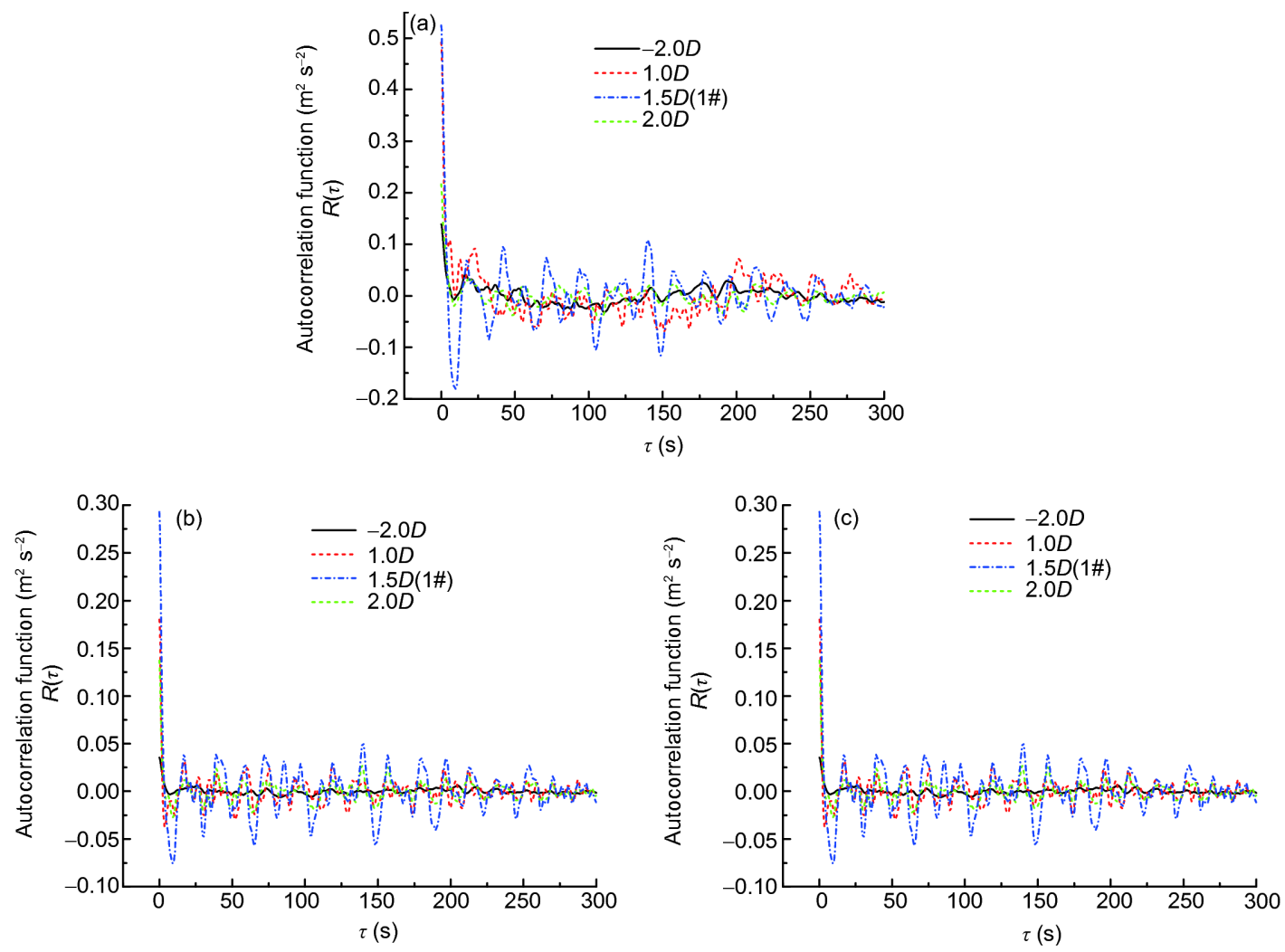

Figure 9 (Color online) Auto-correlations of (a) axial fluctuation velocity $u^{\prime}$, (b) lateral fluctuation velocity $v^{\prime}$, (c) vertical fluctuation velocity $w^{\prime}$. 

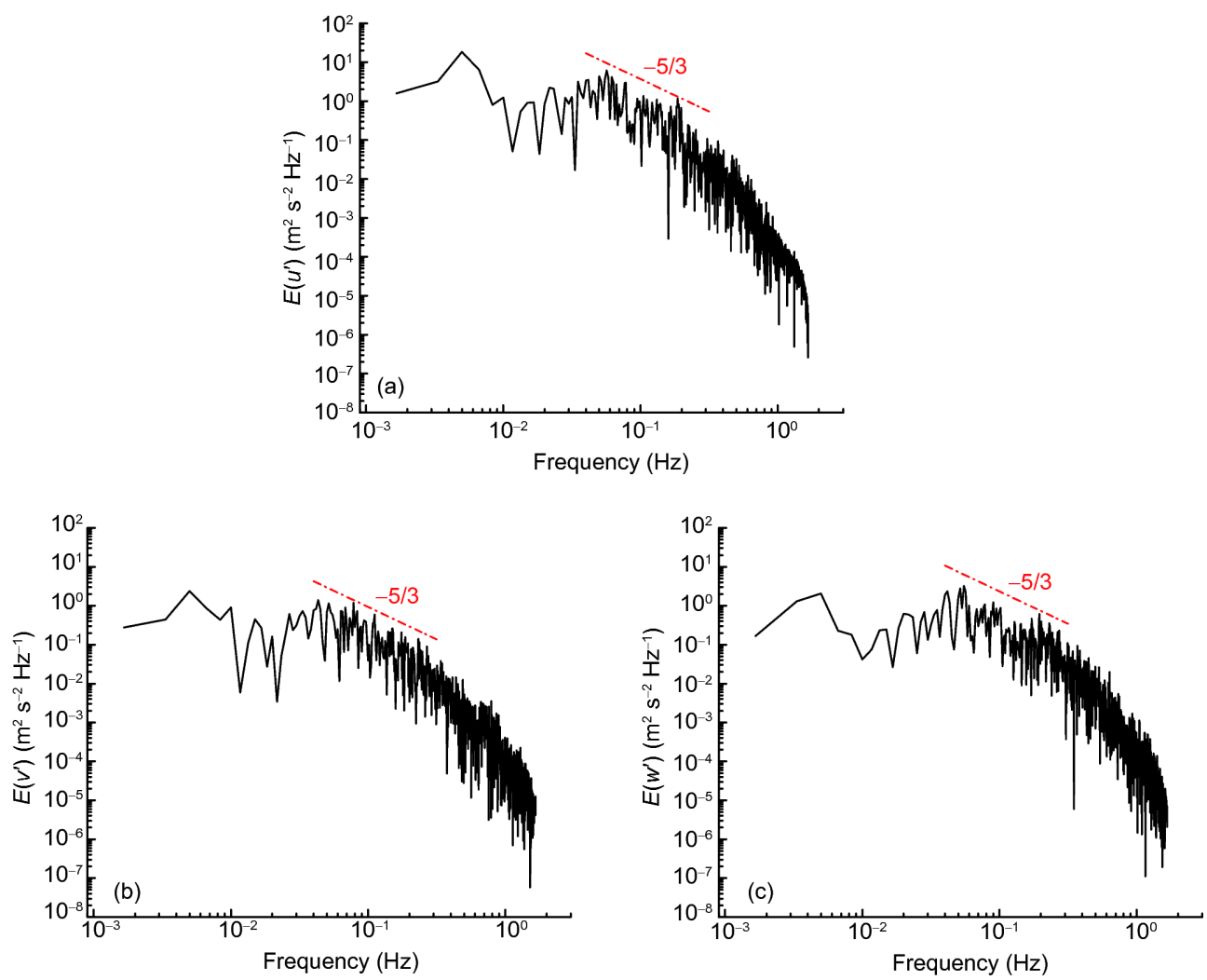

Figure 10 Power spectra of three components. (a) Axial velocity component, $u^{\prime}$; (b) lateral velocity component, $v^{\prime}$; (c) vertical velocity component, $w^{\prime}$.

field is between 0 and 0.45 , so the corresponding wake skew angle $\chi$ should range between $10.60^{\circ}-13.46^{\circ}$. The wake skew angle obtained by LES is about $12^{\circ}$, which agrees well with eq. (12). Figure 11(b) shows the distribution of the wind speed and the tip vortex in the wake flow field. Due to the influence of the yawed rotor, the tip vortex tilts to one side.

\subsection{Time-frequency analysis of wind-turbine loads}

To study the turbulence effects, we analyzed the turbulence kinetic energy (TKE), coherent turbulence kinetic energy (CTKE), fluctuating horizontal flow angle $\left(\psi^{\prime}\right)$, and vertical

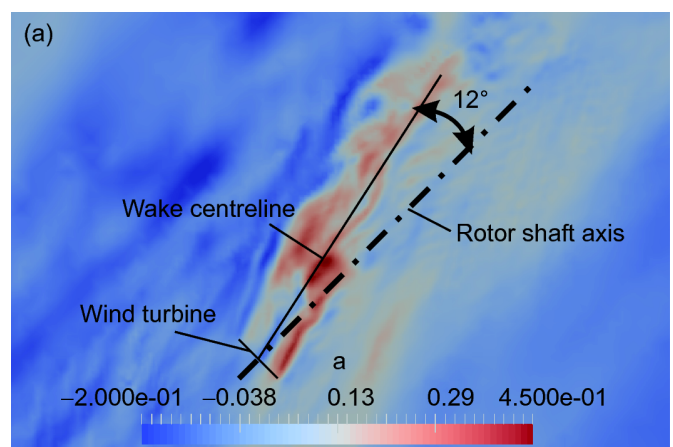

angle $\left(\alpha^{\prime}\right)$ given by eqs. (13)-(16), respectively [43-45].

$$
\begin{aligned}
& \operatorname{TKE}(t)=\frac{1}{2}\left[u^{\prime}(t)^{2}+v^{\prime}(t)^{2}+w^{\prime}(t)^{2}\right], \\
& \operatorname{CTKE}(t)=\frac{1}{2}\left(\left(u^{\prime}(t) v^{\prime}(t)\right)^{2}\right. \\
& \left.+\left(u^{\prime}(t) w^{\prime}(t)\right)^{2}+\left(v^{\prime}(t) w^{\prime}(t)\right)^{2}\right)^{1 / 2}, \\
& \psi^{\prime}(t)=\tan ^{-1}\left[\frac{v^{\prime}(t)}{u^{\prime}(t)}\right], \\
& \alpha^{\prime}(t)=\tan ^{-1}\left[\frac{w^{\prime}(t)}{u^{\prime}(t)}\right] .
\end{aligned}
$$

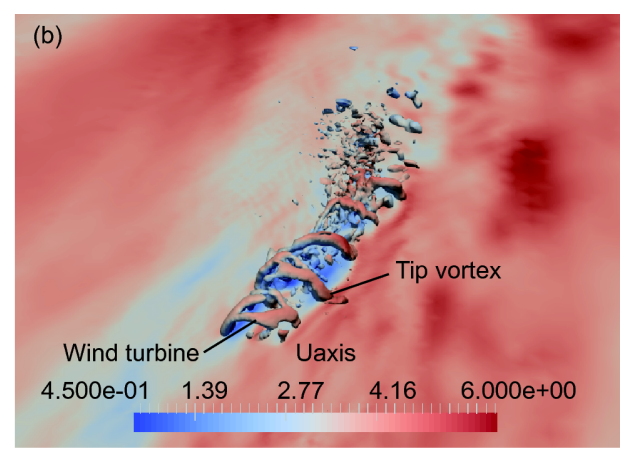

Figure 11 Wake flow field of the wind turbine. (a) Distribution of the axial induction factor $a$ and wake skew angle $\chi$; (b) the distribution of the wind speed and the tip vortex. 


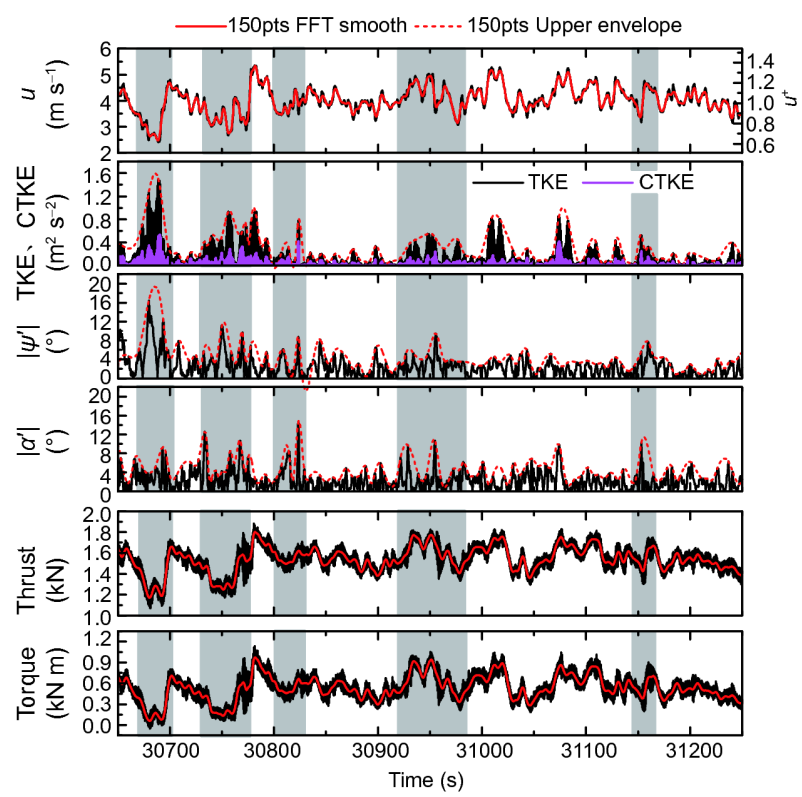

Figure 12 (Color online) Inflow wind speed, TKE and CTKE, variable quantities of fluctuating horizontal and vertical flow angles, power, and thrust of the rotor in $600 \mathrm{~s}$.

The instantaneous TKE value together with CTKE provides a "sensor" of the coherent structures present in the flow. $\psi^{\prime}$ and $\alpha^{\prime}$ reflect the angle changes in the local wind direction. Figure 12 shows the inflow wind speed (row 1), TKE together with CTKE (row 2), absolute values of $\psi^{\prime}$ (row 3 ) and $\alpha^{\prime}$ (row 4), power (row 5), and thrust (row 6) of the rotor in the 600 -s sampling period. When the inflow is in a turbulent state, the power and thrust of the rotor are positively correlated with wind velocity. If we compare rows 1 to 4 , we see that the occurrence of extremum in the TKE and CTKE time series correspond with the trends in the variable quantities of the fluctuating horizontal and vertical flow angles. As such, frequent changes in the inflow wind direction may cause increases in TKE and CTKE, and have an impact on the power and thrust of a wind turbine.

As we can see in Figure 13, there are some peaks at frequencies $1.82 \mathrm{~Hz}\left(2 f_{\mathrm{t}}\right), 3.68 \mathrm{~Hz}\left(4 f_{\mathrm{t}}\right)$, and $5.42 \mathrm{~Hz}\left(6 f_{\mathrm{t}}\right)$, which are multiples of the rotation frequency $\left(f_{\mathrm{t}}=0.91 \mathrm{~Hz}\right)$ of the wind turbine. This reveals that the torque and thrust loads on the blades are unequal and cause an asymmetric load cycle due to the vertical wind shear and the passage of turbulent structures, after which the blade strikes the shaft during one revolution, which increases the fatigue load on the shaft.

\section{Conclusions}

Using wake measurements carried out on a flat ground area in Gansu, China $\left(37^{\circ} 05^{\prime} \mathrm{N}, 103^{\circ} 57^{\prime} \mathrm{E}\right)$, we modeled a $33-\mathrm{kW}$ wind turbine operating in a neutral ABL based on the theories of LES and ALM. A comparison of the LES and experimental results demonstrates the ability of the coupled ALM-LES method to successfully model wind turbines operating in a neutral ABL.

To validate the neutral $\mathrm{ABL}$, we simulated an $\mathrm{ABL}$ case without any wind turbine as a precursor and the results show that the distributions of the potential temperature, average velocity, and fluctuation velocity in the $\mathrm{ABL}$ are consistent with the experimental and theoretical results. Then, we set a $33-\mathrm{kW}$ wind turbine at the center of the flow field with a $10.6^{\circ}$ yaw angle. When the wind is not aligned with the turbine axis, the thrust component normal to the flow direction drives some of the turbulence energy away from the center of the wake. The wake skew angle calculated by LES is consistent with the theoretical results. We then performed an analysis on the auto-correction function and spectral characteristics of the incoming flow and found the region in front of the turbine to be mainly affected by atmospheric turbulence, whereas the region behind the turbine was mainly affected by the wake structure. We performed an FFT of the auto-correlation function to obtain the power spectra and found all the inflow spectra to exhibit a region of $-5 / 3$ slope, which proves the ability of LES to reproduce the energy cascade from larger to smaller scales. Relative to the lateral and vertical velocities, more energy is contained in the power spectrum of the axial velocity, which shows that co-
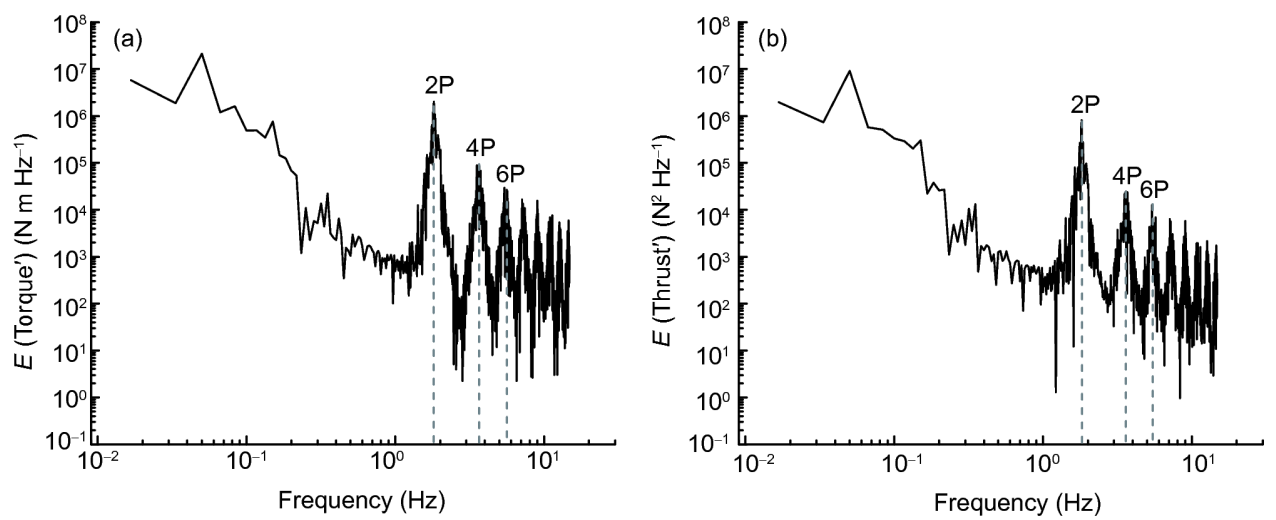

Figure 13 Spectra of (a) torque and (b) thrust. 
herent turbulence structures have more energy in the horizontal direction. To further analyze the wind turbine interaction with the $\mathrm{ABL}$, we performed a joint time-frequency analysis of the incoming flow and the response of the wind turbine. Our analyses of the TKE, CTKE, wind direction, thrust, and power of the wind turbine revealed that frequent changes in the inflow wind direction may cause the TKE and CTKE to increase and have an impact on the power and thrust of a wind turbine. Furthermore, anisotropic wind shear and turbulent structures cause an asymmetric load cycle, which then impose a strike by the turbine blade on the shaft during one revolution and increases the fatigue load on the shaft.

This work was supported by the National Basic Research Program of China (Grant No. 2014CB046201), the National Natural Science Foundation of China (Grant Nos. 51465033, 51766009, and 51479114), the Thousand Talents Program, NSFC-RCUK_EPSRC, the platform construction of ocean energy comprehensive supporting service (2014) (Grant No. GHME2014ZC01), the High-tech Ship Research Projects Sponsored by MIITC Floating Support platform project (Grant No. 201622), and the State Key Laboratory of Ocean Engineering at Shanghai Jiao Tong University.

1 R. Vautard, F. Thais, I. Tobin, F. M. Bréon, J. G. Devezeaux de Lavergne, A. Colette, P. Yiou, and P. M. Ruti, Nat. Commun. 5, 3196 (2014).

2 J. D. Mirocha, B. Kosovic, M. L. Aitken, and J. K. Lundquist, J. Renew. Sustain. Energy 6, 013104 (2014).

3 M. Z. Jacobson, C. L. Archer, and W. Kempton, Nat. Clim Change 4, 195 (2014).

4 H. Lu, and F. Porté-Agel, Phys. Fluids 23, 065101 (2011).

5 S. B. Roy, S. W. Pacala, and R. L. Walko, J. Geophys. Res. 109, D19101 (2004).

6 S. Shamsoddin, and F. Porté-Agel, Bound.-Layer Meteorol. 163, 1 (2017).

7 L. P. Chamorro, and F. Porté-Agel, Bound.-Layer Meteorol. 136, 515 (2010).

8 M. A. Carper, and F. Porté-Agel, Bound.-Layer Meteorol. 126, 157 (2007).

9 M. A. Carper, and F. Porté-Agel, Bound.-Layer Meteorol. 127, 73 (2008).

10 M. Bastankhah, and F. Porté-Agel, Energies 10, 908 (2017).

11 M. J. Churchfield, S. Lee, J. Michalakes, and P. J. Moriarty, J. Turbul 13, N14 (2012).

12 G. España, S. Aubrun, S. Loyer, and P. Devinant, J. Wind Eng. Industrial Aerodyn. 101, 24 (2012).

13 S. Shamsoddin, and F. Porté-Agel, J. Fluid Mech. 837, R3 (2018).

14 R. E. Keck, M. de Maré, M. J. Churchfield, S. Lee, G. Larsen, and H. Aagaard Madsen, Wind Energ. 17, 1689 (2015).

15 M. Abkar, and F. Porté-Agel, Phys. Fluids 27, 035104 (2015).

16 J. Hong, M. Toloui, L. P. Chamorro, M. Guala, K. Howard, S. Riley, J.
Tucker, and F. Sotiropoulos, Nat. Commun. 5, 4216 (2014).

17 M. S. Adaramola, and P. Å. Krogstad, Renew. Energy 36, 2078 (2011).

18 L. A. Martínez-Tossas, M. J. Churchfield, and S. Leonardi, Wind Energ. 18, 1047 (2015).

19 K. Nilsson, S. Ivanell, K. S. Hansen, R. Mikkelsen, J. N. Sørensen, S. P. Breton, and D. Henningson, Wind Energ. 18, 449 (2015).

20 C. Q. Liu, and X. S. Cai, Sci. China-Phys. Mech. Astron. 60, 084731 (2017).

21 Y. Q. Wang, and C. Q. Liu, Sci. China-Phys. Mech. Astron. 60, 114712 (2017).

22 Q. Hu, Y. Li, Y. Di, and J. Chen, J. Renew. Sustain. Energy 9, 064501 (2017).

23 G. Wang, and X. Zheng, J. Fluid Mech. 802, 464 (2016).

24 L. J. Vermeer, J. N. Sørensen, and A. Crespo, Prog. Aerosp. Sci. 39, 467 (2003).

25 F. Porté-Agel, Y. T. Wu, H. Lu, and R. J. Conzemius, J. Wind Eng. Ind. Aerodyn. 99, 154 (2011).

26 M. Calaf, C. Meneveau, and J. Meyers, Phys. Fluids 22, 015110 (2010).

27 A. Jimenez, A. Crespo, E. Migoya, and J. Garcia, Environ. Res. Lett. 3, 015004 (2008).

28 N. Marjanovic, J. D. Mirocha, B. Kosović, J. K. Lundquist, and F. K. Chow, J. Renew. Sustain. Energy 9, 063308 (2017).

29 L. A. Martínez-Tossas, M. J. Churchfield, and C. Meneveau, Wind Energ. 20, 1083 (2017).

30 M. Shives, and C. Crawford, Renew. Energy 92, 273 (2016).

31 M. F. Howland, J. Bossuyt, L. A. Martínez-Tossas, J. Meyers, and C. Meneveau, J. Renew. Sustain. Energy 8, 043301 (2016).

32 J. N. Sørensen, and W. Z. Shen, J. Fluids Eng. 124, 393 (2002).

33 N. Troldborg, J. N. Sørensen, and R. Mikkelsen, J. Phys.-Conf. Ser. 75, 012063 (2007).

34 D. Li, T. Guo, Y. Li, J. Hu, Z. Zheng, Y. Li, Y. Di, W. Hu, and R. Li, Sci. China-Phys. Mech. Astron. doi: 10.1007/s11433-018-9219-y.

35 A. S. Ghate, and S. K. Lele, J. Fluid Mech. 819, 494 (2017).

36 N. Troldborg, Actuator Line Modeling of Wind Turbine Wakes, Dissertation for the Doctoral Degree (Technical University of Denmark, Denmark, 2009), p. 13.

37 C. H. Moeng, J. Atmos. Sci. 41, 2052 (1984).

38 C. M. Rhie, and W. L. Chow, AIAA J. 21, 1525 (1983).

39 I. H. Abbott, and A. E. Von Doenhoff, Theory of Wing Sections. Including a Summary of Airfoil Data (Dover, NewYork, 1959).

40 L. A. Viterna, and R. D. Corrigan, Fixed Pitch Rotor Performance of Large Horizontal Axis Wind Turbines, Technical Report (NASA, 1982).

41 P. K. Kundu, and I. M. Cohen, Fluid Mechanics (Elsevier, Burlington, 2010), pp. 541-564.

42 R. P. Coleman, A. M. Feingold, and C. W. Stempin, Evaluation of the Induced-Velocity Field of an Idealized Helicoptor Rotor, Technical Report (NASA, 1945).

43 J. M. Jonkman, and M. L. Buhl, FAST User's Guide, Technical Report (NREL, 2005).

44 Y. Li, J. H. Yi, H. Song, Q. Wang, Z. Yang, N. D. Kelley, and K. S. Lee, Appl. Phys. Lett. 105, 023902 (2014).

45 B. J. Jonkman, TurbSim User's Guide, Technical Report (NREL, 2009). 